\title{
Effects of Apple Polyphenol on Fat Metabolism in Mice
}

\author{
Ruimin $\mathrm{Fu}^{1,2}$, Wei Tang ${ }^{1}$, Hong Zhang ${ }^{1}$, Huiping Chang ${ }^{1}$, and Wuling Chen ${ }^{2, *}$ \\ ${ }^{1}$ College of health management, Henan Finance University, Zhengzhou, Henan, China \\ ${ }^{2}$ College of Life Science, Shaanxi Normal University, Xi'an, Shaanxi, China
}

\begin{abstract}
To study the regulation effect of apple polyphenol on fat metabolism in mice. The mice were randomly divided into 5 groups, except the basic control group, the other 4 groups were given high fat diet, body weight was measured once a week, and the obesity model was established. The basal control group and the obesity model group were intragastrically given normal saline $(4.2 \mathrm{mg} /(\mathrm{kgbw} . \mathrm{d})$, The three dose groups were gavaged separately with apple polyphenol 4.2, 8.4, $12.6 \mathrm{mg} /(\mathrm{kg}$ bw. d). After 4 consecutive weeks, the body weight of mice, the contents of total cholesterol (TC), total triglyceride (TG), high density lipoprotein cholesterol (HDL-C), low density lipoprotein cholesterol (LDL-C), malondialdehyde (MDA) in serum and the activities of superoxide dismutase (SOD), glutathione peroxidase (GSH-Px), liver lipase (HL) and lipoprotein lipase (LPL) in serum. should be measured respectively. Apple polyphenol can significantly reduce the body weight, TG, LDL-C, TC and MDA contents, coupled with the atherosclerosis index (AI) of obese mice. It can increase the serum HDL-C content of obese mice, and effectively increase the activities of SOD, GSH-Px, HL and LPL in mice. Apple Polyphenol can reduce blood lipid and prevent atherosclerosis via regulating fat metabolism in mice.
\end{abstract}

\section{Introduction}

Apple polyphenol, the general name of polyphenol substances contained in apples has more than 80 pharmacological functions such as preventing hypertension, anti-tumor, anti-aging, anti-mutation, antiradiation and anti-allergy[1]. Therefore, it can be widely used in food, medicine and cosmetics industry. Although there are many studies on the extraction and separation of apple polyphenol and its antioxidant function in vitro in China, there are few studies on the regulation of metabolism in vivo, which to some extent affects the further development and utilization of apple polyphenol. Although it has been reported that apple polyphenol has the functions of weight loss, lipid-lowering and antioxidant in vivo, the mechanism of its action has not been thoroughly studied. Using mice as experimental subjects, the author studied the function of apple polyphenol in lowering blood lipid and preventing atherosclerosis (AS), and revealed the possible mechanism of lowering blood lipid and preventing atherosclerosis from the perspective of regulating liver fat metabolism. The research results will provide a scientific basis for the further development and utilization of apple polyphenol[2-3].

\section{Materials and Methods}

\subsection{Materials and Preparation of Apple Polyphenol}

Apple Polyphenol is homemade in the lab. Kunming mice (body weight $(20.0+2)$ g) were provided by Henan Provincial Center for Disease Control and Prevention. High density lipoprotein cholesterol (HDL-C) kit, triglyceride CTG kit and serum total cholesterol (TC) kit were purchased from Zhejiang Dongfang Genomics Biological Products Co., Ltd. Plus, super-oxide dismutase (SOD) test box, glutathione peroxidase (GSH-Px) test box malondialdehyde (MDA) test box, liver lipase (HL) and lipoprotein lipase (LPL) test box were purchased from Shanghai Shifeng Biotechnology Co., Ltd.

The apple should be heated and then dried and crushed at low temperature. Acetone solution with volume fraction of $90 \%$ is extracted twice with ultrasound assisted extraction according to a certain solid-liquid ratio. The crude product is obtained after filtration, concentration and freeze-drying of the extracted solution[4].Separation and purification of crude products were conducted refer to literature [5-6].

\subsection{Establishment of Diet-induced Obesity Mice Model}

Mice were fed with basal diet[7] in the animal chamber to observe their adaptation to the environment. According to 
body weight, they were randomly divided into 5 groups with 10 rats in each group. Among them, the basic control group was fed with normal diet: Mice in obesity model group and 3 different dose groups were fed high fat diet [The mass fraction was $80 \%$ basic feed, $0.5 \%$ cholesterol, $6.5 \%$ lard, $15 \%$ egg yolk powder, $0.2 \%$ cholate]. Feeding for 15 days, free eating and drinking water, feeding once a day in the morning, weighing once a week.

\subsection{Grouping and administration of animals}

After the establishment of obesity model[8] mice, each group was fed a basal diet. Gastric administration at 9:00 every day: Apple Polyphenol low-dose group (4.2mg/ $\mathrm{kGBW} / \mathrm{d}$ ), medium-dose group (8.4mg/ kGBW/ d), highdose group (12.6mg/ kGBW /d). Basal control group and obesity model group were given normal saline $(4.2 \mathrm{mg} /$ $\mathrm{KGBW} / \mathrm{d}$ ) for 28 consecutive days. The amount of gavage was adjusted according to body weight, and food and water were freely consumed during gavage.

\subsection{Measurement Method}

The body weight of mice was measured once a week at the same time. After 4 weeks, eye blood was collected and serum was separated to determine the contents of TG, TC, HDL-C, SOD, GSH-Px and MDA respectively.

\subsection{Statistical treatment}

SPSS22.0 software should be used for processing and significance analysis, and the data results were expressed as mean \pm standard error.

\section{Result and analysis}

\subsection{Establishment results of feed induced obesity mouse model}

The body weight of mice was significantly increased after 2 weeks of feeding, and the body weight of the 4 high fat diet groups was more significantly increased than that of the basic control group. Eight rats were randomly selected from the high-fat diet group. Blood samples were collected from the eyeballs, and serum was separated to measure the contents of TG and TC. TG content was (3.88 $\pm 0.18) \mathrm{mmol} / \mathrm{L}$, greater than the critical value of $1.7 \mathrm{mmol} / \mathrm{L}$; TC content was $(7.88 \pm 0.68) \mathrm{mmol} / \mathrm{L}$, higher than the critical value of $6.58 \mathrm{mmol} / \mathrm{L}$, indicating that the model of obesity induced by high fat diet was successfully prepared.

\subsection{Effects of apple polyphenol on body weight and food efficiency in mice}

The effects of apple polyphenol on body weight and food efficiency of mice were shown in Table 1.

As shown in Table 1, at the beginning of the experiment, there was no significant difference in the body weight of mice between the experimental groups $(\mathrm{P}>0.05)$. At the end of the experiment, the body weight of apple polyphenol high-dose group was significantly lower than that of obesity model group $(\mathrm{P}<0.05)$, but there was no significant difference compared with the basic control group $(\mathrm{P}>0.05)$. Compared with the basic control group and the obesity model group, there was no significant difference in the net gain of body weight in the other experimental groups $(\mathrm{P}>0.05)$.

Table 1 Effects of apple polyphenol on quality and food efficiency in mice

\begin{tabular}{cccccc}
\hline Groups & Initial mass & Final mass & Net additive mass & $\begin{array}{c}\text { Total quality of } \\
\text { intake }\end{array}$ & Food utility rate \\
\hline Basal control group & $18.89 \pm 0.68$ & $28.98 \pm 1.67^{\mathrm{a}}$ & $10.18 \pm 0.16$ & $170.29 \pm 4.81$ & $6.12 \pm 0.09$ \\
Obesity model group & $21.68 \pm 0.79$ & $34.67 \pm 1.89$ & $13.52 \pm 0.62$ & $169.49 \pm 5.21$ & $7.88 \pm 0.19$ \\
low-dose group & $21.28 \pm 0.58$ & $32.58 \pm 1.26$ & $11.28 \pm 0.19$ & $170.12 \pm 6.33$ & $5.69 \pm 0.78$ \\
Middle dose group & $21.32 \pm 0.81$ & $31.31 \pm 1.68$ & $10.89 \pm 0.18$ & $167.49 \pm 8.43$ & $6.63 \pm 0.71$ \\
High dose group & $20.49 \pm 0.49$ & $29.23 \pm 0.89^{\mathrm{a}}$ & $9.41 \pm 0.29^{\mathrm{a}}$ & $164.99 \pm 7.58$ & $5.59 \pm 0.68$ \\
\hline
\end{tabular}

Notes: Compared with the obesity model group, the difference was significant; b.Compared with the obesity model group, the difference was extremely significant; c.Compared with the basic control group, the difference was significant. d.The difference was significant compared with the basal control group.

With the increase of intragastric dose, the body weight of mice decreased gradually, indicating that apple polyphenol could inhibit the growth of body weight of experimental mice. With the increase of the amount of intragastric administration, the inhibitory degree of body weight increased, and the high dose group had the best inhibitory effect on the increase of body weight. Amid the experiment, there was no significant difference in the average food utilization rate between the experimental groups $(\mathrm{P}>0.05)$, indicating that different doses of apple polyphenol and high-fat feed did not affect the food utilization rate of the experimental animals.

\subsection{Effects of apple polyphenol on blood lipids}

The effects of apple polyphenol on TC and TG in mice are shown in Table 2.

As shown in Table 2, TG and TC in the basic control group were significantly different from those in the obesity model group and the apple polyphenol dose group 
$(\mathrm{P}<0.01)$, which fully demonstrated the success of the obesity model modeling. There was a significant difference in TG level between apple polyphenol dose group and obesity model group $(\mathrm{P}<0.01)$. With the increase of the dose of apple polyphenol, the TG level increased, indicating that there was a dose-effect relationship between apple polyphenol and the decrease of TG level, and within this range, the low dose of apple polyphenol group had the best effect of reducing TG level. Compared with the obesity model group, the serum TC level of apple polyphenol dose group showed a decreasing trend, and there was a very significant difference between the apple polyphenol dose group and the obesity model group $(\mathrm{F}<0.01)$. With the increase of the dosage of apple polyphenol, Tc showed a decreasing trend, indicating that the higher the dosage of apple polyphenol, the better the effect of reducing TC. The results showed that apple polyphenol can reduce the TG and TC in blood of mice, which is helpful to regulate blood lipid.

Table 2 Effects of apple polyphenol on TC and TG in mice

\begin{tabular}{ccc}
\hline Groups & $\begin{array}{c}\text { TG } \\
\text { Content/(mmol/ } \\
\mathrm{L})\end{array}$ & $\begin{array}{c}\text { TG } \\
\text { Content } /(\mathrm{mmol} / \\
\mathrm{L})\end{array}$ \\
\hline $\begin{array}{c}\text { Basal control group } \\
\text { Obesity model }\end{array}$ & $0.68 \pm 0.03$ & $6.06 \pm 0.13$ \\
group & $1.68 \pm 0.08$ & $2.90 \pm 0.36$ \\
Low-dose group & $1.16 \pm 0.17^{\mathrm{bc}}$ & $1.68 \pm 0.38^{\mathrm{b}}$ \\
Middle dose group & $1.20 \pm 0.06^{\mathrm{bd}}$ & $1.36 \pm 0.27^{\mathrm{bd}}$ \\
High dose group & $1.22 \pm 0.16^{\mathrm{bd}}$ & $1.28 \pm 0.32^{\mathrm{bd}}$ \\
\hline
\end{tabular}

Notes: a. Compared with the obesity model group, the difference was significant; $b$. Compared with the obesity model group, the difference was extremely significant; $c$. Compared with the basic control group, the difference was significant. d. The difference was significant compared with the basal control group..

\subsection{Effects of apple polyphenol on HDL-C,LDL-C and $\mathrm{Al}$ in mice}

The effects of apple polyphenol on HDL-C,LDL-C and AI in mice are shown in Table 3.HDLC and LDLC of apple polyphenol dose groups were significantly different from those of obesity model group $(\mathrm{P}<0.01)$. HDL-C and LDL$\mathrm{C}$ of Apple Polyphenol groups were significantly higher than those of the basic control group $(\mathrm{P}<0.05)$, indicating that apple polyphenol can effectively increase the content of HDL-C, reduce the content of LDL-C. In addition, Apple polyphenol had a dose-effect relationship to increase the content of HDL-C, that is, HDL-C showed a decreasing trend with the increase of the dosage, and the low-dose group had the best effect on increasing the content of HDL-C. Besides, there was a very significant difference in AI between the obesity model group and the basic control group $(\mathrm{P}<0.01)$, which further indicated that the obesity-induced modeling was successful and the atherosclerosis was achieved. Compared with the obesity model group, the AI of the apple polyphenol dose group showed a decreasing trend, and there was a very significant difference between the apple polyphenol dose group and the obesity model group $(\mathrm{P}<0.01)$. Plus, apple polyphenol showed a dose-effect relationship to decrease AI, that is, AI showed an upward trend with the increase of Apple polyphenol dose. In this range, the low-dose group had the best effect on reducing atherosclerosis.

Table 3 Effects of apple polyphenol on HDL-C,LDL-C and AI in mice

\begin{tabular}{cccc}
\hline Groups & $\begin{array}{c}\text { HDL-C } \\
\text { Content } \\
/(\mathrm{mmol} / \mathrm{L})\end{array}$ & $\begin{array}{c}\text { LDL-C } \\
\text { Content/(mm } \\
\text { ol/L) }\end{array}$ & AI \\
\hline $\begin{array}{c}\text { Basal control } \\
\text { group }\end{array}$ & $0.56 \pm 0.02^{\mathrm{a}}$ & $1.36 \pm 0.15^{\mathrm{b}}$ & $1.65 \pm 0.23^{\mathrm{b}}$ \\
$\begin{array}{c}\text { Obesity } \\
\text { model group } \\
\text { Low-dose } \\
\text { group }\end{array}$ & $0.18 \pm 0.06^{\mathrm{c}}$ & $2.25 \pm 0.28^{\mathrm{d}}$ & $18.25 \pm 3.26^{\mathrm{d}}$ \\
$\begin{array}{c}\text { Middle dose } \\
\text { group }\end{array}$ & $0.69 \pm 0.14^{\mathrm{bc}}$ & $0.42 \pm 0.21^{\mathrm{bc}}$ & $0.46 \pm 0.12^{\mathrm{bd}}$ \\
$\begin{array}{c}\text { High dose } \\
\text { group }\end{array}$ & $0.66 \pm 0.09^{\mathrm{bc}}$ & $0.33 \pm 0.01^{\mathrm{bc}}$ & $0.79 \pm 0.08^{\mathrm{bd}}$ \\
\hline
\end{tabular}

Notes: a. Compared with the obesity model group, the difference was significant; $b$. Compared with the obesity model group, the difference was extremely significant; $c$. Compared with the basic control group, the difference was significant. d. The difference was significant compared with the basal control group.

\subsection{Effects of apple polyphenol on antioxidant enzyme activity and MDA content in blood of mice}

The effects of apple polyphenol on antioxidant enzyme activity and MDA content in the blood of experimental mice are shown in Table 4.

As shown in Table 4, SOD in apple polyphenol groups was significantly different from that in obesity model group $(\mathrm{P}<0.01)$. There was also significant difference between the apple polyphenol dosage groups and the basic control group $(\mathrm{P}<0.05)$, indicating that the apple polyphenol dosage could effectively improve the activity of SOD. Compared with obesity model group, the activity of GSH-Px in apple polyphenol groups was significantly increased $(\mathrm{P}<0.01)$. The activity of GSH-Px increased with the increase of the dose, indicating that apple polyphenol could effectively improve the activity of GSHPx enzyme in mouse serum, and the higher the dose, the better the effect. Apple Polyphenol could effectively reduce the content of MDA in serum of mice. There was a significant difference in MDA content between the lowdose and medium-dose groups and the obesity model group $(\mathrm{P}<0.01)$, and there was a very significant difference in MDA content between the high-dose group and the obesity model group $(\mathrm{P}<0.01)$. With the increase of apple polyphenol dose, the content of MDA showed a decreasing trend, indicating that the content of MDA in serum of mice could be effectively reduced in each apple polyphenol dose group, and the higher the dose, the better the effect. 
Table 4 Effects of apple polyphenol on antioxidant enzyme activity and MDA content in blood of mice

\begin{tabular}{|c|c|c|c|}
\hline Groups & SOD Vitality/(U/mL) & GSH-px Vitality/(U/mL) & MDA Content $(\mathrm{nmol} / \mathrm{mL})$ \\
\hline Basal control group & $96.79 \pm 0.01^{\mathrm{b}}$ & $14.99 \pm 1.15^{\mathrm{b}}$ & $3.78 \pm 0.67^{\mathrm{a}}$ \\
\hline Obesity model group & $76.98 \pm 4.99^{\mathrm{ab}}$ & $12.27 \pm 2.28^{\mathrm{a}}$ & $7.78 \pm 0.38^{\mathrm{bc}}$ \\
\hline Low-dose group & $171.33 \pm 8.19^{\mathrm{bd}}$ & $25.31 \pm 0.22^{\mathrm{bc}}$ & $6.45 \pm 0.79^{\mathrm{ab}}$ \\
\hline Middle dose group & $430.62 \pm 15.14^{\mathrm{bd}}$ & $26.42 \pm 1.52^{\mathrm{bd}}$ & $6.36 \pm 0.31^{\mathrm{ab}}$ \\
\hline High dose group & $505.78 \pm 9.18^{d}$ & $28.36 \pm 0.72^{\mathrm{bd}}$ & $4.56 \pm 0.21^{\mathrm{b}}$ \\
\hline
\end{tabular}

Notes: Compared with the obesity model group, the difference was significant; b.Compared with the obesity model group, the difference was extremely significant; c.Compared with the basic control group, the difference was significant. d.The difference was significant compared with the basal control group.

\section{Discussion}

A large number of studies have confirmed that lipid metabolism disorders are closely related to atherosclerosis, and lipid changes include changes in the contents of HDL, TG, TC, LDL, etc. Ans the severity of atherosclerosis increased linearly with the increase of plasma cholesterol level, there was a positive correlation between plasma cholesterol concentration and coronary heart disease mortality. Elevated LDL-C level can be the most important risk factor for atherosclerosis and cardiovascular and cerebrovascular diseases caused by atherosclerosis.

SOD and GSH-Px constitute the main antioxidant enzyme system in the body, which can reduce the generation of reactive oxygen free radicals and prevent the damage period caused by lipid peroxides to the body tissues. In addition, apple Polyphenol is known as the seventh nutrient after dietary fiber in Japan. Its antioxidant and free radical scavenging capacity is 50 times that of VE and 20 times that of VC. At present, there are few experimental studies on the regulation of apple polyphenol in vivo, and its mechanism is still unclear. It is found that apple polyphenol can effectively reduce the body weight of mice, reduce the serum TC, TG and LDL-C content, it can reduce weight and lipid, can improve the activity of SOD, inhibit the generation of lipid peroxidation product MDA, and has an antioxidant effect. From the perspective of regulating fat metabolism, this study studied the function and possible mechanism of apple polyphenol to reduce weight and reduce lipid and prevent atherosclerosis. It showed that apple polyphenol can reduce serum TC, TG and LDL-C content, increase HDL-C content, and reduce $\mathrm{AI}$ index. It has the function of lowering blood lipid and preventing atherosclerosis. And then, apple polyphenol can not only increase SOD activity and reduce MDA content, but also increase the activity of GSH-Px.

In conclusion, apple polyphenol can reduce the content of TC, TG and LDL-C in serum by increasing the activity of antioxidant enzymes SOD and GSH-Px in the body, Furthermore, it can promote the generation of HDL-C, reduce the deposition of lipids in the intima of blood vessels, increase free radical scavenging and increase the body's antioxidant capacity, and prevent the occurrence and development of atherosclerosis by regulating fat metabolism. Moreover, apple polyphenol is extracted from the common edible fruit apple,so it is expected to become a new and widely used health food to regulate blood lipid and prevent atherosclerosis.

\section{Funding}

This work was financially supported by the state scholarship fund (China Scholarship Council Program No.10006), Key Teachers Training Program of Henan Higher Education Institutions(2020GGJS265), Key scientific research of Institution of higher learning in Henan Province(21B180002), and Henan Provincial Science and Technology Project (182102310995, and 182102110002).

\section{References}

1. Vuoso, D.C., D’Angela, S., Ferraro, R., Caserta, S., Guido, S., Cammarota, M., Porcelli, M., Cacciapuoti, G.(2020) Annurca apple polyphenol extract promotes mesenchymal-to-epithelial transition and inhibits migration in triple-negative breast cancer cells through ROS/JNK signaling. Scientific reports, 10: 117.

2. Cong, L., Bremer, P., Mirosa, M.(2020) Functional Beverages in Selected Countries of Asia Pacific Region: A Review. Beverages, 6: 21.

3. Varaeva, Y.R., Livantsova, E.N., Polenova, N.V., Kosyura, S.D., Nikitjuk, D.B., Starodubova, A.V.(2020) Characteristics of blood lipid profiles of professional athletes: a literature review. Current pharmaceutical design, 26: 98-102.

4. Wu K.H., Ho, C.T., Chen, Z.F., Chen, L.C., Peng J.W., Lin, T.N., Ho, Y.S.(2017) The apple polyphenol phloretin inhibits breast cancer cell migration and proliferation via inhibition of signals by type 2 glucose transporter. Journal of Food and Drug Analysis, 26: 221-231.

5. Li, D., Cui, Y., Wang, X.J.,Liu, F., Li, X.L.(2021) Apple polyphenol extract alleviates lipid accumulation in free-fatty-acid-exposed HepG2 cells via activating autophagy mediated by SIRT1/AMPK signaling. Phytotherapy Research, 35: 1416-1431.

6. Zhang, X.H., Wang, Y.P., Jiang, W., Mao, R.(2020) Effect of expanded shrub litter on decomposition of graminoid litter in a temperate freshwater marsh. Plant and Soil, 451: 409-418. 
7. Xiang, J.H., Qin, L., Zhao, D., Xiong, F., Wang, G.T.,Zou, H., Li, W.X., Li, M., Song, K.B., Wu, S.G.(2019) Growth performance, immunity and intestinal microbiota of swamp eel (Monopterus albus) fed a diet supplemented with house fly larvae (Musca domestica). Aquaculture Nutrition, 26: 693-704.

8. Alansari, W.F., Eskandrani, A.A.(2020) The Anticarcinogenic Effect of the Apple Polyphenol Phloretin in an Experimental Rat Model of Hepatocellular Carcinoma. Arabian Journal for Science and Engineering, 45: 4589-4597. 